\title{
Nomenclature in Kuiper Belt
}

\author{
Brett Gladman \\ Observatoire de la Côte d'Azur, B.P. 4229, 06304 Nice Cedex 4, France
}

\begin{abstract}
Given the advances in our understanding of the dynamical structure in the Kuiper Belt since 1990, this paper re-examines nomenclature issues regarding dynamical structures in the belt.
\end{abstract}

\section{Orbital groupings}

The orbital structure of the trans-neptunian region of our Solar System preserves valuable clues regarding planet formation. We begin by describing orbital groupings in this region (Fig. 1); as well as discussing regions of orbital parameter space which have as yet no known objects.

(1) The classical belt, between 40 and $48 \mathrm{AU}$. The 'classical belt' (Jewitt et al. 1998, JLT 98 hereafter) is so-named because it most resembles the Kuiper Belt that was originally searched for: a belt of dynamically cold (low $e$ and $i$ ) objects outside Neptune representing the left-over planetesimal disk which never succeeded in accreting into planetary-sized bodies. Gravitational erosion over the lifetime of the Solar System (Duncan et al. 1995) is responsible for clearing out certain regions of the belt, in particular the prominent gap between 40 and $42 \mathrm{AU}$. The eccentricity distribution is truncated by the Neptune-approaching limit of pericenters $q=a(1-e)<35 \mathrm{AU}$, and objects that may have once been above this line (Fig. 1b) were eliminated by gravitational perturbations; therefore the inclination distribution is more primordial and gives a better measure of the dynamical perturbation of the region. However, the inclination distribution of the detected objects (Fig. 1a) is biased towards low inclinations (Jewitt et al. 1996) because Trans-Neptunian Object (TNO) surveys focus on the ecliptic, where TNOs on inclined orbits spend the smallest fraction of their time.

(2) Plutinos are those TNOs trapped, like Pluto, in the 3:2 mean-motion resonance with Neptune at $39.5 \mathrm{AU}$. Some plutinos have $q<35 \mathrm{AU}$ (Fig. 1b), but the resonance prevents close encounters (see, e.g., Malhotra 1996). Higher eccentricities produce Uranus crossing, truncating the distribution at $e \sim 0.4$. The resonance's interior is stable over the age of the Solar System, although Morbidelli (1997) showed that plutinos with large libration amplitude escape by dynamical diffusion on gigayear time scales. There are other TNOs in resonances throughout the region (Morbidelli et al. 1995, Malhotra 1996) with similar dynamical properties, which can collectively be called the 'resonant population' (JLT98), although we single out the 2:1 for special consideration below. 
(3) TNOs with $a=36-39$ AU and small $e$ and $i(<0.05)$ are predicted to be stable by Duncan et al. (1995), with some stable regions at $i \geq 20^{\circ}$. Prior to mid-1999, no TNOs except for $1995 \mathrm{DA}_{2}$ (protected in the 4:3 resonance) were known to be in this region, potentially implying that the dynamical processes that sculpted the region left nothing in this small stable region of phase space. However, $1998 \mathrm{SN}_{165}$ and $1998 \mathrm{HN}_{151}$ are now in this region; should it be considered part of the 'classical belt', though separated from it by the $2: 3$ resonance and the unstable $40-42 \mathrm{AU}$ region?
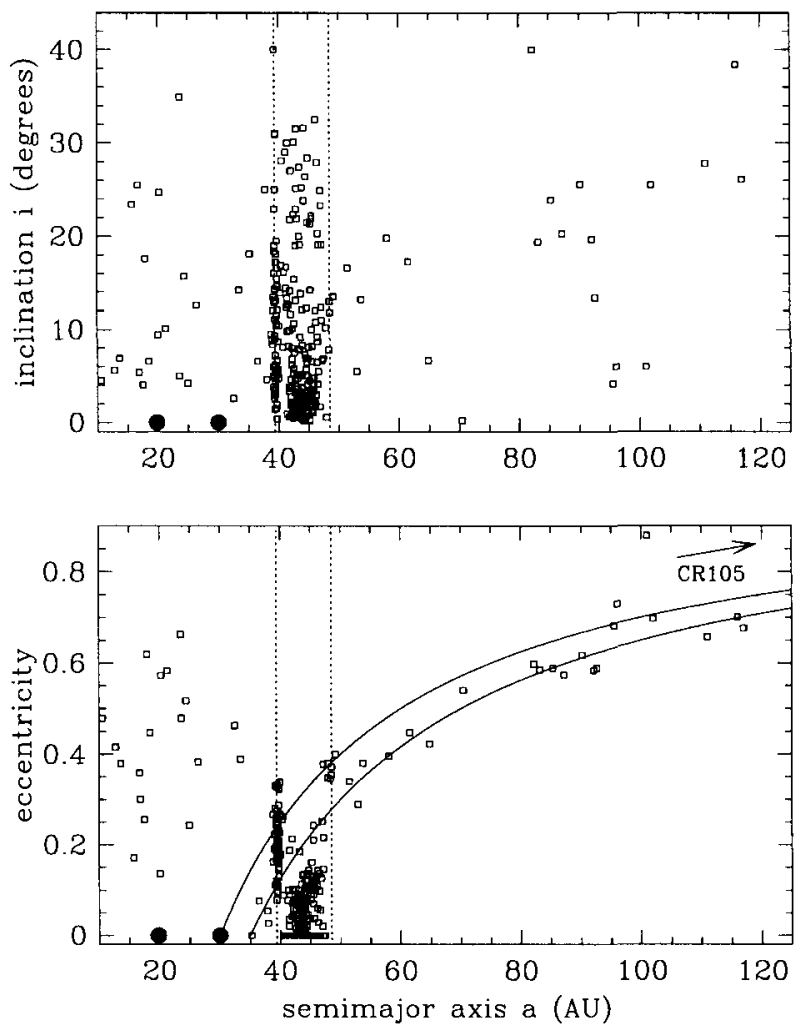

Figure 1. The orbital distribution of TNOs in the MPC database, as of July 3,2000 . All orbits have been included (even those with as little as 24-hour arcs). For reference, we show the distances of Uranus and Neptune (heavy dots), vertical lines at the 3:2 (39.4 AU) and 2:1 (47.8 AU) resonances with Neptune, and curves in the bottom panel marking perihelion distances of 30 and $35 \mathrm{AU}$.

(4) Objects in the 2:1 resonance, at $a=47.8 \mathrm{AU}$, were also not identified until early 1999. There are now several objects whose current orbit estimates place them near the resonance, but only $1998 \mathrm{SZ}_{10}$ appers to be firmly lodged 
within it. Being the most distant first-order mean-motion resonance, and figuring prominently is some formation scenarioes, the $2: 1$ serves as natural division between the 'inner' Kuiper Belt and the 'outer' Kuiper Belt.

(5) Low-e TNOs with $a>48$ AU do not exist in the current Minor Planet Center (MPC) orbital database. Even in 1998 there were objects whose orbits penetrated the region beyond $48 \mathrm{AU}$, and thus there was never any question that TNOs existed outside this heliocentric distance; the issue was whether flux-limited surveys with their bias to the nearest objects should have found them. Dones (1997) and JLT98 modelled available data and concluded that surveys should have detected such objects if they existed. Gladman et al. (1998) found a steeper luminosity function, and showed that the non-detection of objects outside $\Delta \sim 50$ AU was not significant. Since the beginning of 1999, 10 objects have been detected outside of a distance of $48 \mathrm{AU}$, but it is not known if they have semimajor axes outside $48 \mathrm{AU}$ and/or low eccentricities, but none can yet be proven to be so.

(6) Scattered disk objects are loosely defined as those having highly eccentric orbits outside Neptune. Objects populating this structure could have either escaped from the Kuiper Belt to Neptune-encountering orbits, or have been emplaced there primordially as the giant planets cleared the outer Solar System of left-over planetesimals. The first such object identified was $1996 \mathrm{TL}_{66}$, but many of the TNOs discovered before 1998 and now lost may have been on highly-eccentric orbits.

(7) Centaurs inside $30 \mathrm{AU}$. The Centaurs represent the transition population between the short-period comets and their source region outside of 30 AU. Modern terminology puts the Centaurs in to the 'ecliptic comet' population (Levison 1996), whose flattened orbital distribution indicates a non-spherical source. For a Kuiper Belt source, proposed supply mechanisms include longterm dynamical instabilities or collisions. Or, it is possible that the 'scattered disk' is sufficiently populated at the current epoch that its continuing dynamical erosion is capable of producing the required input of Jupiter-family comets and Centaurs (Duncan \& Levison 1997). An important recent advance is the identification of two objects, $1999 \mathrm{OX}_{3}$ and $1998 \mathrm{BU}_{48}$, which are the first 'transition objects' between the TNO population and the Centaur population; both are Neptune-crossing with $a>30$ AU but are on orbits not dynamically protected from Neptune. Are these to be classified as Centaurs?

\section{Future Kuiper-Belt nomenclature}

The essential problem is that several terms, including 'scattered disk', are either loosely defined, or dependent on the formation scenario one has in mind.

The term 'scattered disk' seems to trace back to Torbett (1989), who noted that such a structure would form when planetesimals were scattered out from the Uranus-Neptune region. Duncan and Levison (1997) realized that some TNOs escaping the Kuiper Belt onto Neptune-crossing orbits would enter this structure; because their simulations used particles escaping from the Kuiper 


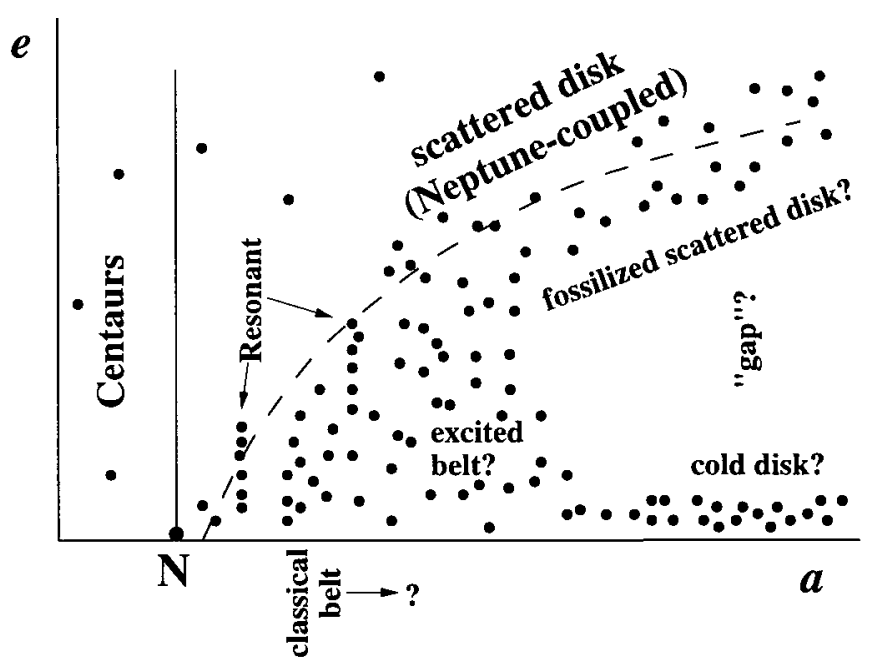

Figure 2. A cartoon showing various orbital classes in the outer Solar System. Centaurs end at $a=30 \mathrm{AU}$. We prefer a dynamically-meaningful definition of the scattered disk related to having the objects currently coupled to Neptune (see text). The resulting boundary is not a strict line on an $(a, e)$ diagram, and is thus shown as a dashed line, dividing it from the classical belt and/or fossilised scattered disk. The cold disk, fossilized scattered disk, and excited belt may or may not exist and depend on whether a 'gap' is found in the $e$ distribution at large semimajor axes, a signature of scattering off a very large body closer to the Sun. 'Resonant' objects include plutinos, but not low-e objects interior to $39 \mathrm{AU}$. The 'classical belt' begins at $40 \mathrm{AU}$ according to the JLT98 definition, but there seems to be no reason why the low-e region interior to $39 \mathrm{AU}$ should be excluded, nor is it clear where (or if) the 'classical belt' should end.

Belt via dynamical diffusion as input, the entire 'scattered disk' thus built is evidently produced by scattering with Neptune. Trujillo et al. (2000) define the scattered disk population as $q \equiv 34-38 \mathrm{AU}$, with the proviso that Neptunescattering is only 'thought' to be its origin. The MPC's current definition seems to be any object on a high-e orbit exterior to orbit of Neptune with $q<38$ or $40 \mathrm{AU}$, although this perihelion limit, and the inner border with the Centaur population, are ill-defined.

Unlike in dynamical simulations, one does not know the past history of observed TNOs; there are multiple dynamical paths or formation scenarios that can produce a TNO on a given orbit. Concretely, consider a TNO at high $e$ in or near the 2:1 resonance, with $q$ near $36 \mathrm{AU}$, where dynamical lifetimes are $\sim 4.5$ Gyr. Duncan and Levison (1997) discuss examples of particles which scatter off Neptune and later find themselves removed from Neptune-crossing in external mean-motion resonances (this is a temporary state, but may last a very long time); such a particle is clearly 'scattered' when looking at its history. However, a TNO could start near $48 \mathrm{AU}$ at lower $e$ and migrate 'up' to higher $e$ in the resonance due to dynamical diffusion over the lifetime of the Solar System (Nesvorný and Roig 2000), and thus this particle has never 'scattered' 
off of anything! If the resonance swept up the body and pushed it to large $e$, as in the Malhotra (1996) scenario, one has another case in which the particle has never been 'scattered'.

One option would be to carve up orbital space into somewhat arbitrary but precisely-defined regions, much like the Apollo/Amor/Aten definitions for the near-Earth asteroids (NEAs). Although the dynamical distinction between the borders of these classes is somewhat arbitrary, they do have the virtue of being clearly defined, and dynamical classes in the NEA region are difficult to define over Gyr time scales. The latter problem is not as serious in the outer Solar System, and thus the following proposal attempts to class TNOs according to the current behaviour.

Objects on planet-crossing orbits with $a<30$ AU remain Centaurs, but with an $a=30$ upper boundary emplaced (Fig. 2); the inner edge of the Centaur distribution presumably occurs where the Jupiter-family comets begin (Levison 1996). This preserves the idea that the Centaurs are the planet-crossing objects between the giant planets. One would then like to call 'Neptune-coupled comets' those TNOs with $a>30 \mathrm{AU}$ whose orbits are coupled with the planet via close encounters, but the term 'scattered disk' is so heavily ingrained in the literature that one can simply hone this term to: 'scattered disk objects' are those which have encounters with Neptune able to move its semimajor axis by $1 \mathrm{AU}$ on Gyr timescales. This definition has dynamical meaning which can be tested via numerical integration (already required to designate an object as resonant), and defines scattered particles via their current dynamical state rather than their past history. It also allows for protected regions: resonant populations can rise above any arbitrary $q$ line, and the low- $e$ region between 36 and 39 AU which might otherwise appear in a definition of the 'scattered disk', is also excluded. It allows the 'transition objects' with $a>30 \mathrm{AU}$ but $q<34 \mathrm{AU}$ to be classed in the scattered population. It also avoids the nomenclature problem that some objects with large $e$ and $q=35-38$ AU may very well not be having close encounters with Neptune, and thus should not be classified as 'scattered objects'. Preliminary dynamical simulations show that many TNOs classified as 'scattered' on the MPC pages never encounter Neptune when integrated for $4 \mathrm{Gyr}$; a very unsatisfactory state of affairs. This definition of scattered disk has weaknesses due to the chaotic behaviour of meta-stable orbits, but is free of many of the holes of other definitions and is not based on assumptions about orbital histories and/or formation scenarios.

Having such a definition of the 'scattered disk' then allows a precise definition of which TNOs are not in it and thus what the 'Kuiper Belt' is! Otherwise one could be faced with a situation, in a stellar passage scenario for example (Ida et al. 2000), where one might consider the whole Kuiper Belt to be the scattered disk...or vice versa...! The Kuiper Belt clearly has sub-components such as the 'resonant objects' and what is currently called the 'classical belt'. The latter is in hindsight a bit of a misnomer because the 'hot' state of this region shows it to be anything but the untouched thin relic of the protoplanetary disk; we prefer the term 'cold disk' for this latter structure if and when it is ever found. Other 'components' of the Kuiper Belt may or may not exist, and observations in the coming years will be needed to resolve the issue. The identification of a 'cold disk' would require proving that a definite separation occurs between it and the 
scattered disk at higher $e$ (Fig. 2), with potentially a 'fossilized scattered disk' (Thommes et al. 2000) also being present.

\section{References}

Dones, L. 1997, in From Stardust to Planetesimals, Y.J. Pendleton \& A.G.G.M. Tielens Eds., ASP Conf. Proceedings 122, 347

Duncan, M., Levison, H., Budd, S.M. 1995, AJ, 110, 3073

Duncan, M., Levison, H. 1997, Science, 276, 1670

Gladman B., Kavelaars, J.J., Nicholson, P., Loredo, T., Burns, J.A. 1998, AJ, 116,2042

Ida, S., Larwood, J., Burkert, A. 2000, ApJ, 528, 351

Jewitt D., Luu J., Chen J. 1996, AJ, 112, 1225

Jewitt D., Luu J., Trujillo C. 1998, AJ, 115, 2125 (JLT98)

Levison, H. 1996, in Completing the Inventory of the Solar System, ASP Conf. Proceedings, 107, 233

Malhotra, R. 1996, AJ, 111, 504

Morbidelli, A., Thomas, F., Moons, M. 1995, Icarus, 118, 322

Morbidelli, A. 1997, Icarus, 127, 1

Nesvorný, D., Roig, F. 2000, Icarus, in press

Thommes, E., Duncan, M., Levison, H. 1999, Nature, 402, 635

Torbett, M.V. 1989, AJ, 98, 1477

Trujillo C., Jewitt D., Luu J. 2000, ApJ Lett., 529, L103 